\title{
Meaning and structural dynamics in poetry: a computational perspective
}

\author{
Alex Gomez-Marin \\ Instituto de Neurociencias (CSIC-UMH), Alicante, Spain \\ E-mail:agomezmarin@gmail.com
}

\begin{abstract}
This work addresses Sri Aurobindo's mantric poem, Savitri, with a computational linguistics approach. This is one of the longest poems ever written in English. We build the connectivity matrix between all main word pairs and analyse its structure. Concepts emerge as directions that better explain the variance of the data in the hyperspace of words. When projected to the low dimensional space of concepts, the vector of attention as the reader moves through the text shows a large correlation across sections of the poem, thus acting the future and the past over again. These findings suggest that the mathematical structure of Savitri is and reflects a substrate for the author's main ideas, facilitating the reader's understanding of the poem's meaning via its long-range dynamical correlations. Acknowledging an irreducible essence to poetry, future studies on the relationship between words and sounds, and sounds and ideas may provide invaluable hints of the origin of language and its intimate relationship with the evolution of human consciousness.
\end{abstract}

\section{INTRODUCTION}

The fact that the insights of this and other pieces of writing are intelligible to you as a reader is perhaps one of the greatest mysteries of language. However, our familiarity with the daily use of words to express ideas is often confused with the understanding of the nature and processes underlying that capacity. Amongst the manifold instances of communication we see in nature, human language is an exceptional case. Although most of the information we often exchange through phones or the internet is a mere exercise of relatedness without much meaningful content, higher expressions of consciousness such as poetry embody complex ideas, from abstract thoughts to very intimate experiences, with an exceptional vividness. How does an idea become text? How is that sign traced back to meaning? How can we almost effortlessly grasp what a book is about despite not remembering the precise word sequence details of any of its thousand pages? Here we tackle these questions using mathematical, statistical, and computational tools. Following procedures developed and applied to prose writings [1], we make a first step towards poetry by analysing how structure supports meaning and how long-range dynamical correlations facilitate understanding.

In this work we concentrate on Sri Aurobindo's Savitri [2]. Savitri is part of a large literary output of more than 30 volumes, most of which are meant to convey the essential substance of Indian spiritual culture. Therefore, language is being used in a very deliberate way. It is based on the Sanskrit oral tradition, which constitutes the basis of a great corpus of linguistic theory. Being one of the longest poems ever written in English literature, Savitri offers an exceptional opportunity to investigate the statistical basis of poetry. Furthermore, the existing translations of Savitri into several languages place it as a privileged system to analyse to what degree the original meaning is conserved in the structure and dynamics across different languages. Most interestingly, Savitri is a mantric 
poem, which poses very interesting questions about the nature, role and differences of speech versus written text. Indeed comparable to Homer's works, this 24,000-line epic and allegorical masterpiece is in the vanguard of the use of quantitative meter [3]. This is a sound-based principle where each line is made of three or four feet in which the most significant word or syllable has more sound-weight-length, conferring the poetry an audible rhythmic structure. As we will discuss in the end, it is essentially in the sound, rather than in the text, where meaning lies. This work deals mainly with text.

\section{RESULTS}

\subsection{The space of words}

The text analysis was based on custom made scripts written in the free programming language $\mathrm{R}$ [4]. Codes are available upon request. For text preparation, hyphens and punctuation marks were removed. All words were ranked according to their number of appearance in the whole text. "Stopwords" were then filtered out. The list of stopwords was minimal, containing only main pronouns, articles, prepositions and adverbs. Words occurring in lower frequencies than a given threshold (in our case less than 12 times in the whole poem) where discarded. No stemming procedures to reduce words to their root were applied here, so that the original inflections and formative elements the author chose were not lost in the analysis.

In numbers, the nearly 24 thousand lines of the poem contain approximately 200 thousand words, out of which 10 thousand are different. This denotes a highly specialised use of language. After filtering for repetitions, stopwords and rare occurrences, we are left with slightly more than 1,600 different main words. Interestingly, most of the words are nouns. In Figure 1, the first 15 words of the main list are shown.

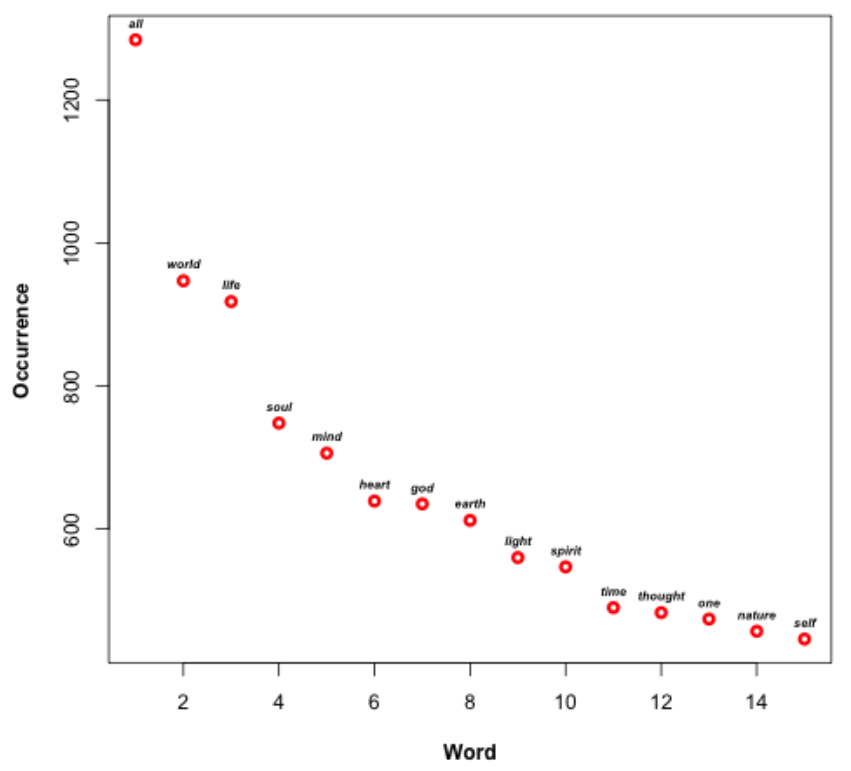

Figure 1. Word occurrences. Number of times each word appears in the whole poem. The plot shows the first 15 words of a total of 1,640 main words, ranked by occurrence, after excluding stop-words and sub-threshold words. Nouns are dominant. The alphabetical list of all words, their occurrence and position in the text is easily obtained by similar means. 


\subsection{Structure}

We calculated the connectivity matrix between all possible pairs of main words within a window of attention. Namely, for every word we counted how many times its pair word is found within a distance of 100 words back and forth; a window that is reasonable for a reader's short-term attention capacities. These pair relationships can be efficiently stored in the so-called co-occurrence matrix, which is then normalised to correct for the absolute frequency of words [1]. Beyond single word statistics, this quantification captures a word-to-word interaction network beyond nearest neighbours. As depicted in Figure 2, the information across one column of the matrix provides hints about how a particular word is related to the rest.

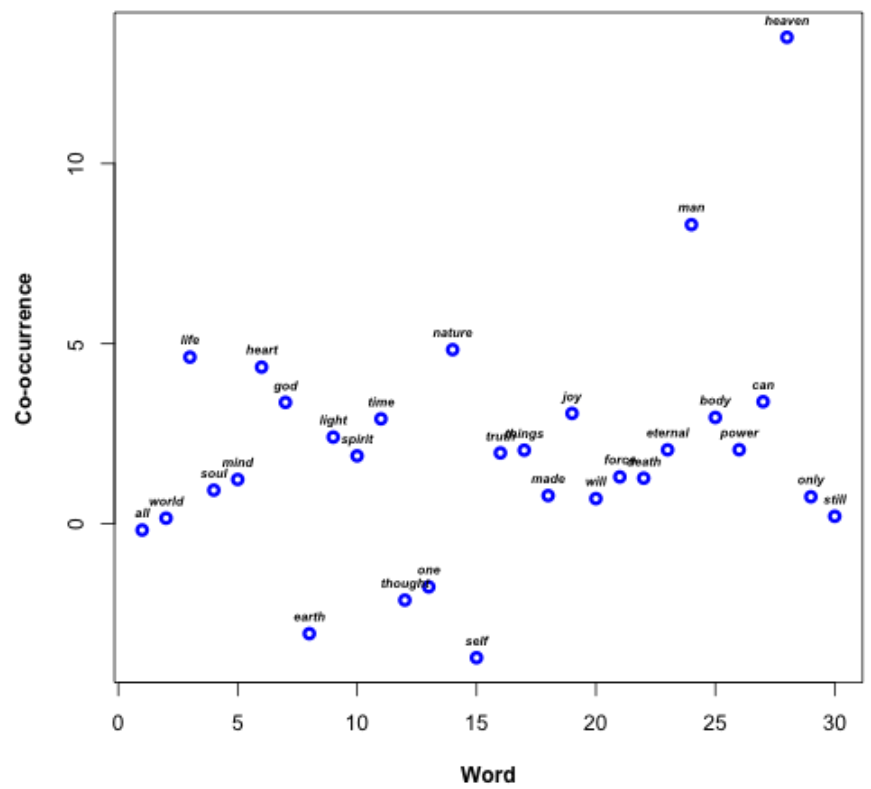

Figure 2. Co-occurrence. Pairwise relationship between the word "earth" (ranking position 8) and each of the 30 words with greater occurrence. Co-occurrence between two words is calculated within a window of attention of 200 words and then normalised for absolute word pair frequencies. Note that in the example shown here "earth" is anti-correlated with itself and with the word "self", its presence is noninformative of the word "all", and it is positively correlated with "heaven" and "man". Namely, "earth" and "heaven" are statistically entangled; they appear close to each other more frequently than random.

Next, in Figure 3 we show Savitris co-occurrence matrix for the first 30 positions, out of its total of 1,640 dimensions, given space visualisation constraints. Note the great compression of information that such a representation allows. 


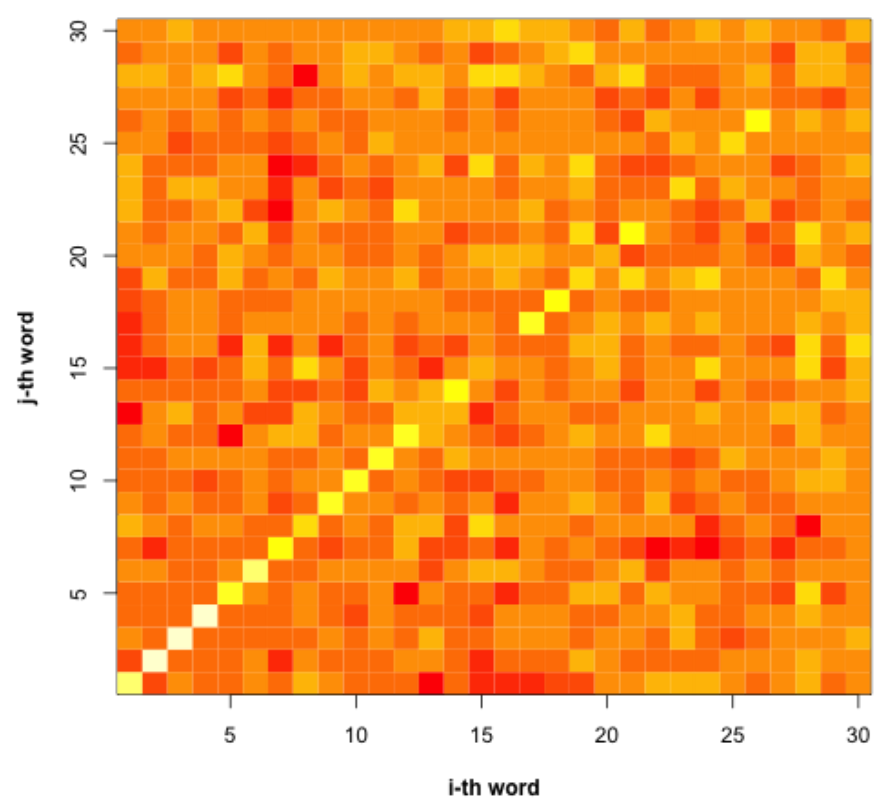

Figure 3. Structure. Matrix quantifying the relative co-occurrence of each word pair. Row 8 corresponds to Figure 2. Red-orange and yellow-white colours depict positive and negative values, respectively. The full matrix is a proxy for the structure of the whole poem, which can be visualised as a data cloud in a large dimensional space. Here we display only the first 30 main words.

\subsection{Dimensions}

The hundred-fold reduction in the number of words we are dealing with is still not a simple enough description to gain an understanding of the richness and complexity of the poem as a whole. Rather than focusing on a particular word or word pair, we aim for a comprehensive measure of the text structure that correlates with meaning; one where only a few dimensions are needed and relevant: we are looking for the emergence of concepts. Then, to learn something interesting beyond the connectivity matrix of the poem, we can ask the following question: given that every line of the matrix is a dimension in a space of words, and given that every column is a data point in that hyperspace, if we think of the poem as a complex cloud of data, is there a reduced set of directions that explains most of the variance in that cloud?

In fact, we are asking two questions: how many directions do we need and what do they mean? The answer to the first will tell us whether the system allows for a dimensionality reduction. For instance, in a two-dimensional space, a simple visual inspection would tell us that an ellipse-like cloud of points can be described by its major and minor axes. Our brain immediately rotates the gestalt and perceives its length and width. For most matters, we do not need to know the position of each and every point anymore, but just to consider both axes. We reduce the swarm of points to two relevant numbers and we know what such directions mean. In four dimensions the same operation is nearly impossible to visualise.

As for the poem, we are dealing with more than a thousand of these. Fortunately, geometric transformations in arbitrarily high dimensional spaces are dealt with at ease using basic algebra. We apply the so-called singular value decomposition to the co- 
occurrence matrix in order to retrieve principal components and singular values, namely, meaningful directions and their relevance. Singular values ranked in decreasing order are shown in Figure 4. To progress with our analysis of Savitri, next we focus on the principal directions with highest singular values.

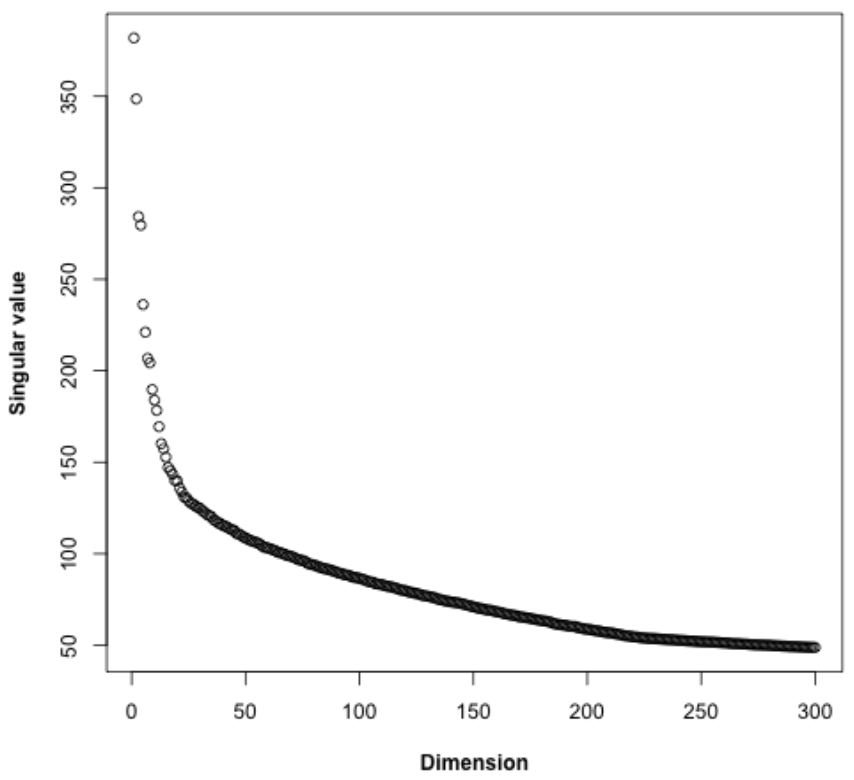

Figure 4. Dimensionality reduction. Singular values, decreasingly ranked, resulting from the singular value decomposition of the co-occurrence matrix. The first values account for most of the variance and so we are interested in their corresponding directions. Both the static and the dynamic features of the poem will be projected to this low dimensional space of ideas.

\subsection{Concepts}

By treating the poem as data within a computational approach, can we gain some understanding? After the dimensionality reduction, we search for meaning along the principal directions. Every principal direction is a vector of coefficients associated to each word. Coefficients close to zero indicate that the corresponding word is not relevant across the direction under consideration. Therefore, words that are either on the positive or negative axis along each principal direction are clusters representative for that concept. We show Savitr's three main concepts below. Regardless of whether these mathematically obtained units of meaning are coincident with the reader's individual experiences or thoughts about Savitri, it is obvious that these are not a collection of random words. Without introducing any semantic bias, meaning may emerge from context in terms of these word clusters extracted by the computer.

The highest singular components from the singular value decomposition of the cooccurrence matrix are the most explanatory directions in the space of words, and can be interpreted as concepts $(\mathrm{C})$ emerging from the poem's structure. For every concept, we show the words that cluster more toward positive $(\mathrm{C}+)$ and negative $(\mathrm{C}-)$ extremes along each direction, based on their coefficients in the singular component. Although copulas are missing, such word combinations indeed capture relevant content, ideas or topics of the poem. 
The first 3 principal "concepts" extracted from the mathematical decomposition are:

C1+: truth, force, man, must, matter, ignorance, law

C1 -: joy, beauty, bright, skey, sweet, happy, Satyavan

C2+: all, mind, thought, self, sense, space, sight

C2 -: earth, death, man, love, fate, pain, grief

C3+: god, earth, light, truth, heaven, buman, bliss

C3 -: night, void, darkness, buge, dead, nothingness, black

These are the highest singular components from the singular value decomposition of the co-occurrence matrix. They are the most explanatory directions in the space of words, and can be interpreted as concepts emerging from the poem's structure. For every concept, we show the words that cluster more toward positive and negative extremes along each direction, based on their coefficients in the singular component. Such word combinations indeed capture relevant content, ideas or topics of the poem.

\subsection{Dynamics}

So far we have not considered the dynamics of the poem, but only its static structural relationships. A poem, and more generally any book, is read in a sequential manner from a beginning to an end. How does the focus of attention of the reader move in the meaningful space of concepts that has emerged? Defining the so-called vector of attention as in [1], we quantify the point in the hyperspace of words where the reader is at every moment in time, assuming he/she can remember the words covered in the previously defined window of attention. In essence, as one goes through the lines of the poem, a different short sequence of words is explored. As shown in Figure 5, we can now ask what concepts the reader's attention is projecting to.

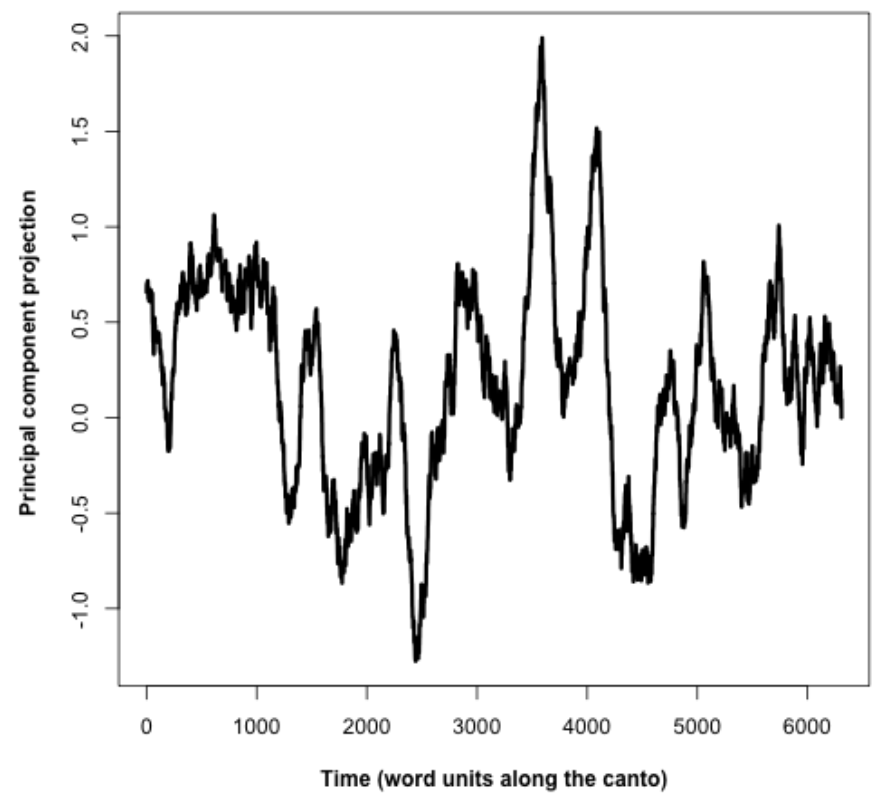

Figure 5. Dynamics. Temporal dynamics of the vector of attention projected on the first concept (C1) as a reader moves through Canto 3 of Book 1, from the beginning to the end. Particular sections of the Canto explore clusters of the concept, while others seem unrelated. 


\subsection{Correlations}

The dynamics uncovered above may prove useful to cluster certain pieces of text by the ideas they explore, and even to summarise them without the need of human intervention. Dwelling upon the space of concepts, we may also uncover the intentions of the author to guide the reader through certain patterns that better convey the story. This approach also allows us to address to what degree and in what way the author's ideas can be recalled and remembered across the whole poem. Why do we have a feeling of continuity in meaning from beginning to end? Issues about memory can be quantitatively measured through temporal correlations. Accordingly, we transformed the whole poem into a long vector of attention and then projected it onto the five main concepts.

We then calculated the auto-correlation function at different times, measured in word distances. Figure 6 demonstrates that correlations are maintained at very long distances. Intriguingly, the corresponding exponent of decay is very low. Strong dynamical correlations are present throughout Savitri, facilitating the limited capacities of the reader to hold on to Sri Aurobindo's transmission of ideas for very long periods of time.

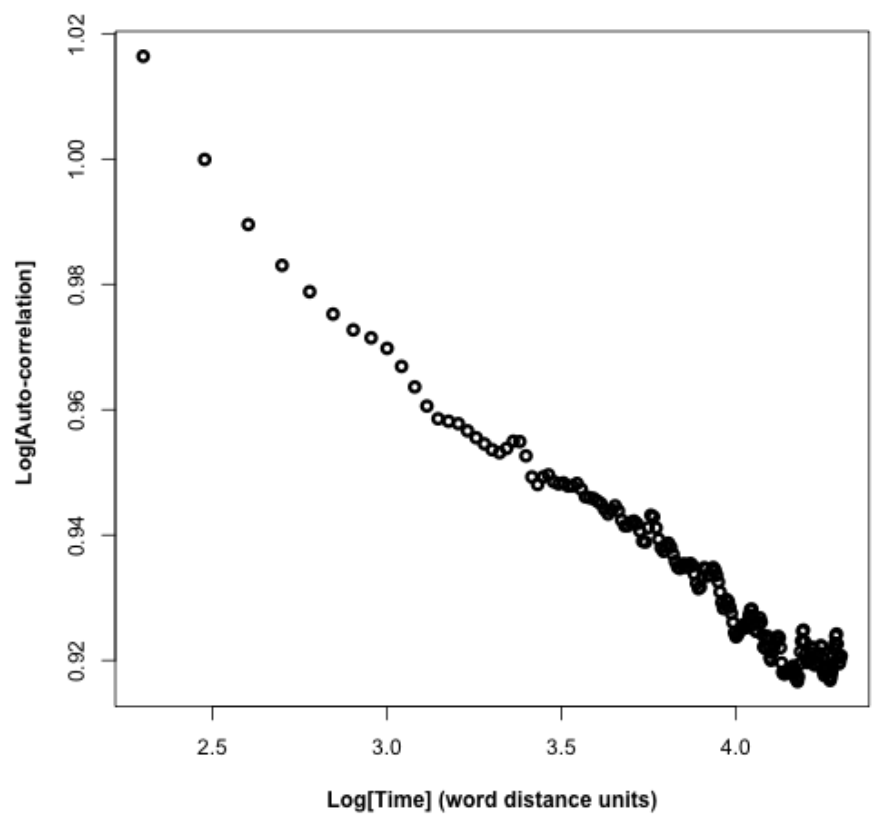

Figure 6. Correlations. Autocorrelation function of the vector of attention of the whole poem, when projected onto the five main concepts, as a function of time understood as distance measured in word units. The linearly decreasing dependence in logarithmic scale implies the existence of long-range correlations: concepts within a window of attention of 200 words are maintained beyond four orders of magnitude, namely, across 10,000 words. Such power law behaviour in written texts has been shown to emerge from an underlying hierarchical organisation [1]. 


\section{DISCUSSION}

\subsection{Summary}

We have illustrated the uses and limitations of a radical dimensionality reduction from the nearly 200 thousand words Savitri consists of to a handful of computationally derived word clusters that may represent its main concepts, therefore potentially revealing the author's ideas in the structure of the text. From such a structure we have then explored the dynamics of reading, finding that within a limited span of attention the poem's principal ideas are correlated across very long distances, which may contribute to the reader's understanding of the complex and multidimensional nature of the thoughts embedded in the text. As previously shown for written text in prose [1], hierarchical structures induce long-range dynamical correlations in written texts. We have demonstrated a similar behaviour now for poetry. Moreover, this piece of work offers us the possibility to scrutinise literature with computational tools even when the goal of the study is not computational. It also raises interesting concerns about the implications for translations, transcriptions and recitations.

By simple extensions to the available codes we wrote to process Savitri, we could address simple questions more focussed on specific aspects of a text. For instance, to study a particular word and see how often it appears and where. Most document readers and internet browsers allow such tasks already. However, the advantage of doing it ourselves is that we could customise it. Let us elaborate on a potential to-do-list. As for words, we could measure whether the word of interest appears more often at the beginning or towards the end of the line. We dealt with the most used words for statistical purposes. We could do the opposite and study less frequent words and their connection network.

We may want to change our definition for close pairs based on the window of attention, perhaps restricting it to the size of a single line or a paragraph. We could focus on the stopwords themselves. Or one could easily check the difference between words in upper and lower case, which is an aspect we have disregarded so far. Plus, we could count the letters of the words in every line, or concentrate on particular parts of a word. The use of the suffixes "-ness", "-less" and "-ing" are valuable targets. The apparent relevance of nouns over adjectives or verbs is worth following up on as well.

Such a custom-made search engine would make it possible to sort the lines of the poem lines by end words as a way to study rhythm. We could even focus on the use of punctuation marks, and analyse the branching from books to cantos, to sections within each canto and then to paragraphs and sentences within each section. Note that we could ask the computer to rewrite the whole poem removing, modifying or adding words according to our particular demands, and then read it ourselves seeking changes in our experience or understanding of it. As we did our analysis based on words, we could repeat it for lines themselves, thus searching for correlations between other units of information. Cantos of similar content could be classified similarly.

Regarding concepts, we only had time to explicitly show the three main ones, but it would be interesting to explore the rest of the list and potentially study the relevance of a concept and its co-appearance with others. For instance, the fifth principal component contains words invoking a sense of dark/lightness: air, far, half, dim, sky, tread, road, past, vague, hardly and dusk. And concept number seven gathers the following list of frightening words: night, pain, darkness, evil, hell, black, sombre, agony, cells, tortured. 


\subsection{Future directions}

Promising future directions targeting greater aims are discussed next. First, one would study the effect of subtle variations on Savitri, as highlighted in [5]. Note that the vector of attention does not capture the particular order of each word in a line, which is central to poetry and thus should be considered in the future. Similarly, it is an irresistible follow-up to look at the immense collection of works written by Sri Aurobindo, searching for principles and perhaps differences, across his varied styles of writing and wide range of topics. The prolific outcome of the author was very well documented in time. One could perhaps find a quantitative evidence of the evolution of his writings. Actually, Savitri was itself written and edited over decades. Sri Aurobindo wrote several books simultaneously. Can the structure and dynamics of every book match the rest?

The great importance of Savitri makes it a poem that is translated into many languages. This offers an unprecedented opportunity to study the effect, and even quality, of a translation by using all the above measures. What varies and what is universal across languages? For instance, it would be interesting to see what concepts emerge when singular value decomposition is applied to Savitri in English, Catalan or Tamil. And whether the phylogeny of such languages constrains the remarkable properties we found for long-range dynamical correlations. For the first time, we could quantitatively address whether we are lost in translation. How easier is it to comprehend a poem in its original language where the signature of the author is present in every single detail? We succeeded in the computational analysis of the poem without any stemming manipulation, which facilitates the direct analysis for any language regardless of whether WordNet is available.

Sound is not text. As trivial as that can sound (or read), it turns out that the oral aspect of human communication may have underlying principles and processes that are different than those of writing and reading. In fact, old traditions relied on speech rather than on text. Of course one fundamental cause for that is the fact that they could not produce and share documents as we do nowadays. However, the mantric nature of many cultural and spiritual teachings states that sound is a key ingredient for getting the message across; not just as a vehicle but often as the very message itself [6]. And let us not forget that language evolved as spoken language, and only much later writing took place. From a linguistic perspective, phonetics is another important aspect of language which should not be disconnected from semantics. In Figure 7 speech is measured for a few lines of poetry by means of a spectrogram, which quantifies intensity, frequency and duration. Following [7], we checked whether the spectral components of speech had different properties when Savitri is recited by different people, and we used normal speaking as a baseline control. We found no significant differences (data not shown). Much more needs to be done in this direction.

Finally, one could envision correlational studies about the effect of reading or hearing Savitri at the level of individual subjective perception and objective physiological functions such as heart beat and breathing rate, or even brain activity by recording voltage changes through the scalp with electroencephalograph techniques. In that situation, two control experiments could be very revealing. First, to check the differences between reading, reading aloud and listening to poetry. Second, to look for those effects that are independent of explicit intellectual comprehension. Could it be more effective to listen to mantric poetry in a language you don't understand than to read a translation in a language you are familiar with? 


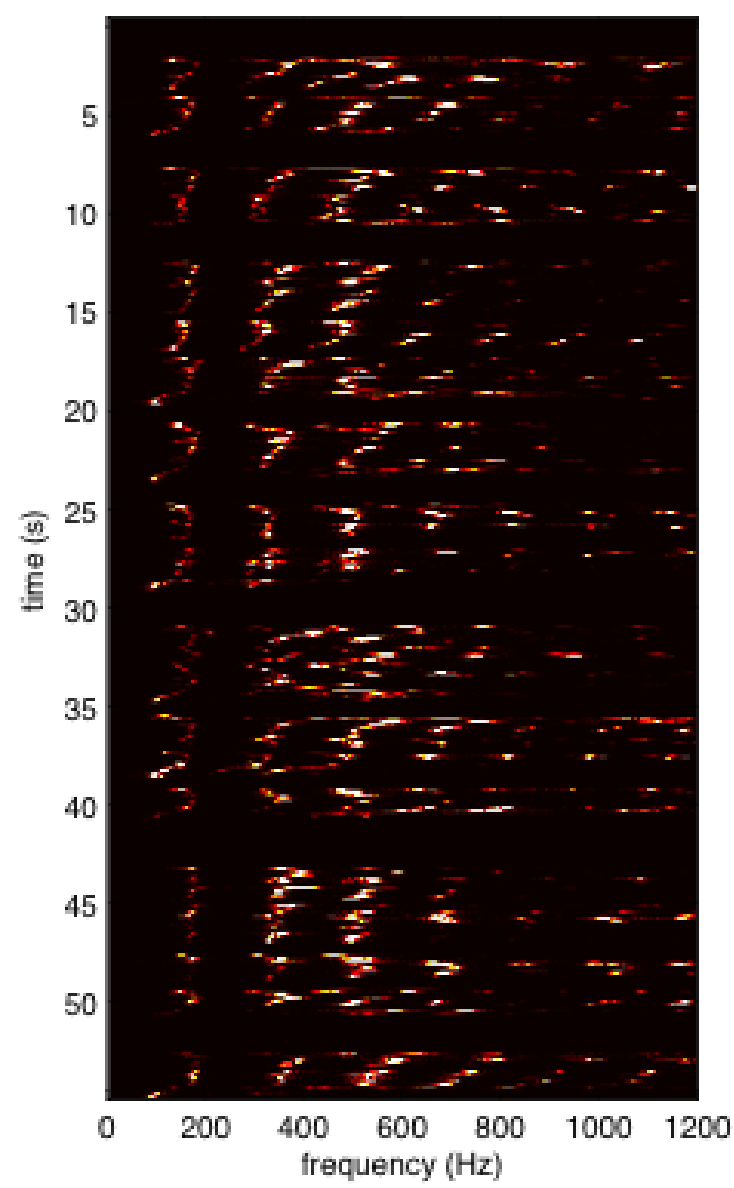

Figure 7. What the ear, but not the eyes, can grasp. Spectrogram graphically depicting measurements of frequency, intensity and duration of lines 1 to 11 of Canto 3 of Book 1 (reading by Rod Hemsell). If a poem is written to be read aloud, and thus heard, it is in the sound, and not in the text, that its secrets are to be found. This kind of quantification could be used in future studies to address the structure and dynamics of meaning transmitted through speech, such as in modern political debates and banal cafe discussions all the way to the ancient oral traditions and mantric poetry.

\subsection{Outlook}

We have mentioned the importance of hierarchical structures in language and their role in long-range correlations [1]. What about the dynamic build-up of complexity in poetry by means of a recursive function? Namely, to what extent a nested repetition that cyclically moves from familiar to unknown states can explain how each part of the poem supports the application of the whole. This would solve, to some degree, what Schopenhauer poses as a contradiction [8]: a single thought must preserve the most perfect unity whereas a book must always have a first and a last line, remaining very unlike an organism, however like one its content may be.

Regarding recursion in a slightly different context, there is an intriguing connection between the evolution of animal behaviour and that of language. First, the relevance of the latter in constraining our understanding of the organisation of animal movement across species [9]; certainly an interesting overlap between linguistics, ethology and neurobiology. Second, recursion, or the capacity to generate an infinite expressive potential from a finite set of elements, as perhaps the only and unique human 
component of the faculty of language in its broad sense [10]. Finally, and bridging the previous two ideas, the fact that the principle of recursion in animal exploratory behaviours, rather than in communication itself, may have been the evolutionary prerequisite for human language to unfold [11].

This offers a provocative hypothesis about the origins of human language as necessarily embodied language, in which the meaning of abstract ideas emerged from sensorimotor metaphors. Playing seriously with words, perhaps that is why to comprehend an idea, or to grasp it, recalls the same action as to firmly hold a thing, namely, to grasp that thing; or why we might say we understand something when are placed under its full influence and power, namely, when we stand under it. To see means both to perceive with your physical eyes as well as to discern or deduce after reflection, expressing comprehension.

Coming back to poetry we may now consider that actions, images and words are intimately intertwined. The poet, while trying to express and impress a whole complex personal experience on the reader, faces a unique and practically impossible task. The reader, failing to understand all that is behind the poet's words, is provided with an image of it, one that is tangible and distinct enough that ideas seem to be extended like objects in space. From this point of view, words and sounds are neither the cause nor the effect of meaning. They are part of it. They form it in terms of space and time by means of images and rhythm, respectively. We dynamically express ourselves by means of words and think in terms of space. The interweaving of our sensorimotor capacities, our conception of time and space, and the unfolding of language is patent.

On the whole, the present work raises many more questions than it answers. This is because of the ill-defined nature of our endeavour, namely, to reduce what is irreducible: the highest expression of human consciousness into a computational problem. This can nevertheless be a departure point from which future studies take over to succeed in opening the doorways of meaning. Nothing prevents us from enquiring about the measurable nature of ideas as conveyed in language forms, despite then being left with nothing but their quantitative correlates devoid of any true qualitative element. Ironically, the very same thing that we had sought to eliminate in the first place, and strove to keep out of the picture during our scientific detour, is precisely what we expect to emerge and what we fail to see fully at the end. Perhaps by attempting such impossible feats we shall become more aware of the necessity to lessen the duality (and the obscure confusion) between quality and quantity. Paraphrasing Bergson [12], it seems that language borrows from text and sound the meaning on which it feeds and restores it to them in the form of a dynamic structure which it has stamped with its own freedom. As the philosopher would put it, instead of seeking to solve the question, we shall show the mistake of those who ask it.

Afterword. Savitri has been written to be heard. It is a whole creation to be experienced, not a piece to be analysed. In this sense, the present work is useless. However, everyone has a particular way to come nearer to Savitri. My hook was the scientific method. At the beginning I spent more time writing code to scrutinize the text than actually immersing myself in the poetry. That was stupid but necessary. Then, by reading it aloud, verse after verse, the rhythm steadily pours on the reader, like droplets on a stalagmite. Thus, one can go from experiment to experience, finally listening quietly with a rather quiet mind. 
Acknowledgements. This research was carried out during a two-months retreat in India. I thank Antim and Shraddhavan for their kind hospitality. I am grateful to Rod Hemsell for pointing me out what the eye cannot get, but only the ear can grasp.

\section{REFERENCES}

[1] E. Alvarez-Lacalle, B. Dorow, J. P. Eckmann, and E. Moses (2006) Hierarchical structures induce long-range dynamical correlations in written texts, Proc. Natl. Acad. Sci. USA 103, 7956-7961.

[2] Sri Aurobindo (1997) Savitri: a Legend and a Symbol, 4th edition, Sri Aurobindo Ashram Trust.

[3] Sri Aurobindo (1997) The Future Poetry, Sri Aurobindo Ashram Trust.

[4] J. Chambers and colleagues (1993) The R Project for Statistical Computing, Bell Laboratories.

[5] R. Y. Deshpande (2012) Man-handling of Savitri, Savitri Foundation.

[6] R. Hemsell (2008) The Poetry of Sri Aurobindo: Mantra, Metrics and Meaning, University of Human Unity Lecture Series.

[7] R. F. Voss, and J. Clarke (1975) 1/f noise in music and speech, Nature 258: 317-318.

[8] A. Schopenhauer (2011) The World as Will and Idea, The Project Gutenberg, Ebook 38427.

[9] Ilan Golani (1992) A mobility gradient in the organization of vertebrate movement: The perception of movement through symbolic language, Behavioural and Brain Sciences 15, 249-308.

[10] M. D. Hauser, N. Chomsky, and W. T. Fitch (2002) The Faculty of Language: What Is It, Who Has It, and How Did It Evolve?, Science 298, 1569.

[11] Ilan Golani, personal communication.

[12] H. Bergson (1988) Matter and Memory, Zone Books, Urzone Inc. 